\title{
Physics and Chemistry of
}

\section{Using Bulk-Like Nanocrystals to Probe Intrinsic Optical Gain Characteristics of Inorganic Lead Halide Perovskites}

\section{Pieter Geiregat' | Jorick Maes' I Kai Chen² I Jonathan De Roo ${ }^{3}$ | Justin Hodgkiss² I Zeger Hens'}

While reports on stimulated emission and lasing by perovskites show great promise, a comprehensive quantification of their optical gain characteristics is lacking. Here, bulk-like colloidal perovskite nanocrystals (NC) are used as a test bed to quantify optical gain by lead halide perovskites. By analyzing colloidal dispersions of such NC, we can deploy a unique combination of broadband transient absorption and ultrafast fluorescence spectroscopy to measure gain coefficients, clarify the gain mechanism, and explore the gain dynamics. Opposite from current literature, we show that optical gain in perovskite NCs is supported by stimulated emission from free carriers and not from excitons or bi-excitons. Importantly, we demonstrate that the concomitant gain coefficients agree with literature results reported for thin films. Finally, we show that, even in the case of fully inorganic lead halide perovskites, a cooling bottleneck hampers the development of net stimulated emission at high excitation density.

\section{Weakly Confined Perovskites}
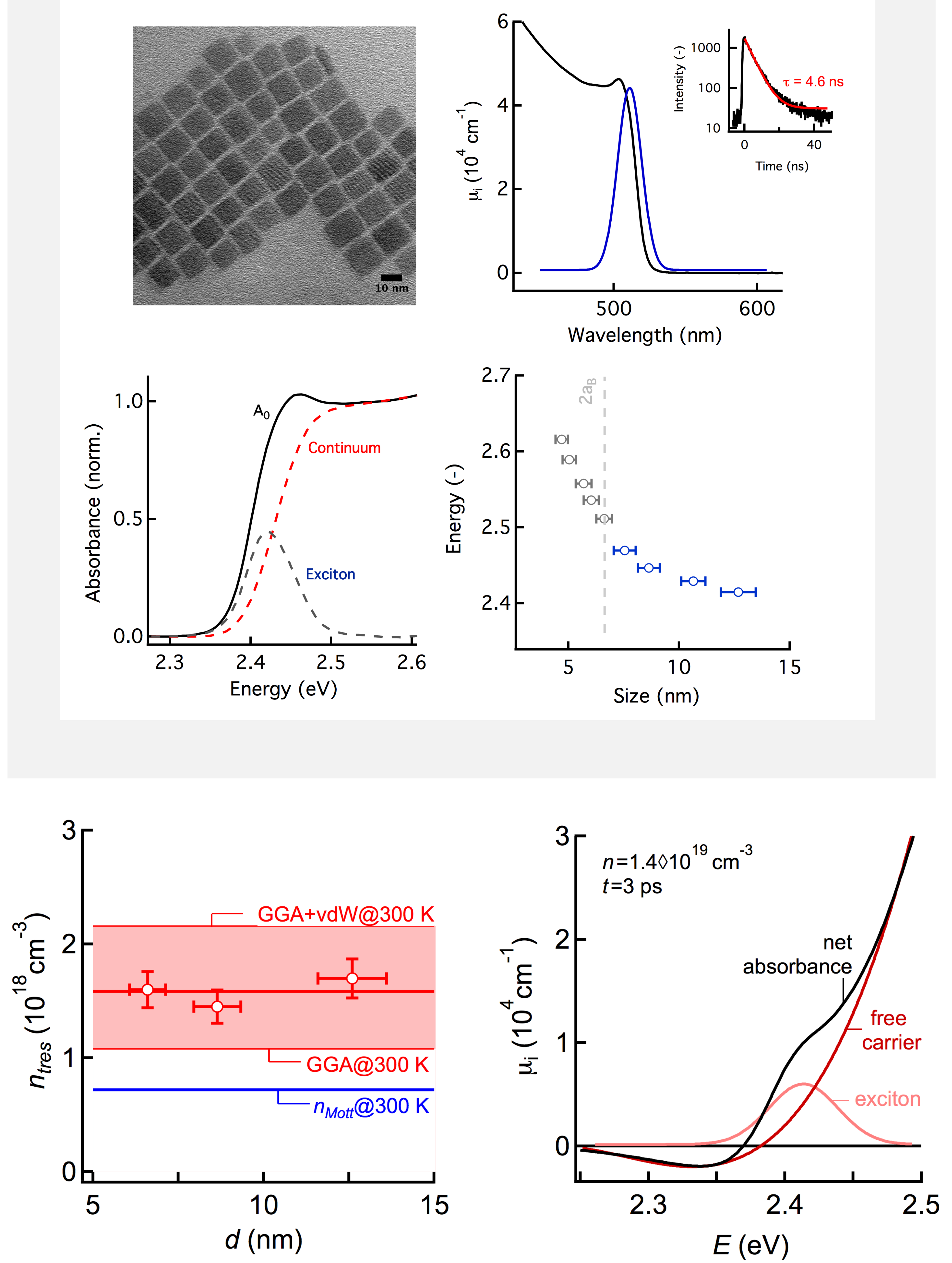

\section{Cooling Bottleneck}

At high density, carrier cooling slows down from a sub-ps to close to 10 ps average phonon emission time. The bottleneck arises from overheated phonon modes and gives rise to the observed reduction in maximum material gain for off-resonant pumping.

\section{Gain Spectroscopy with Varying Pump}
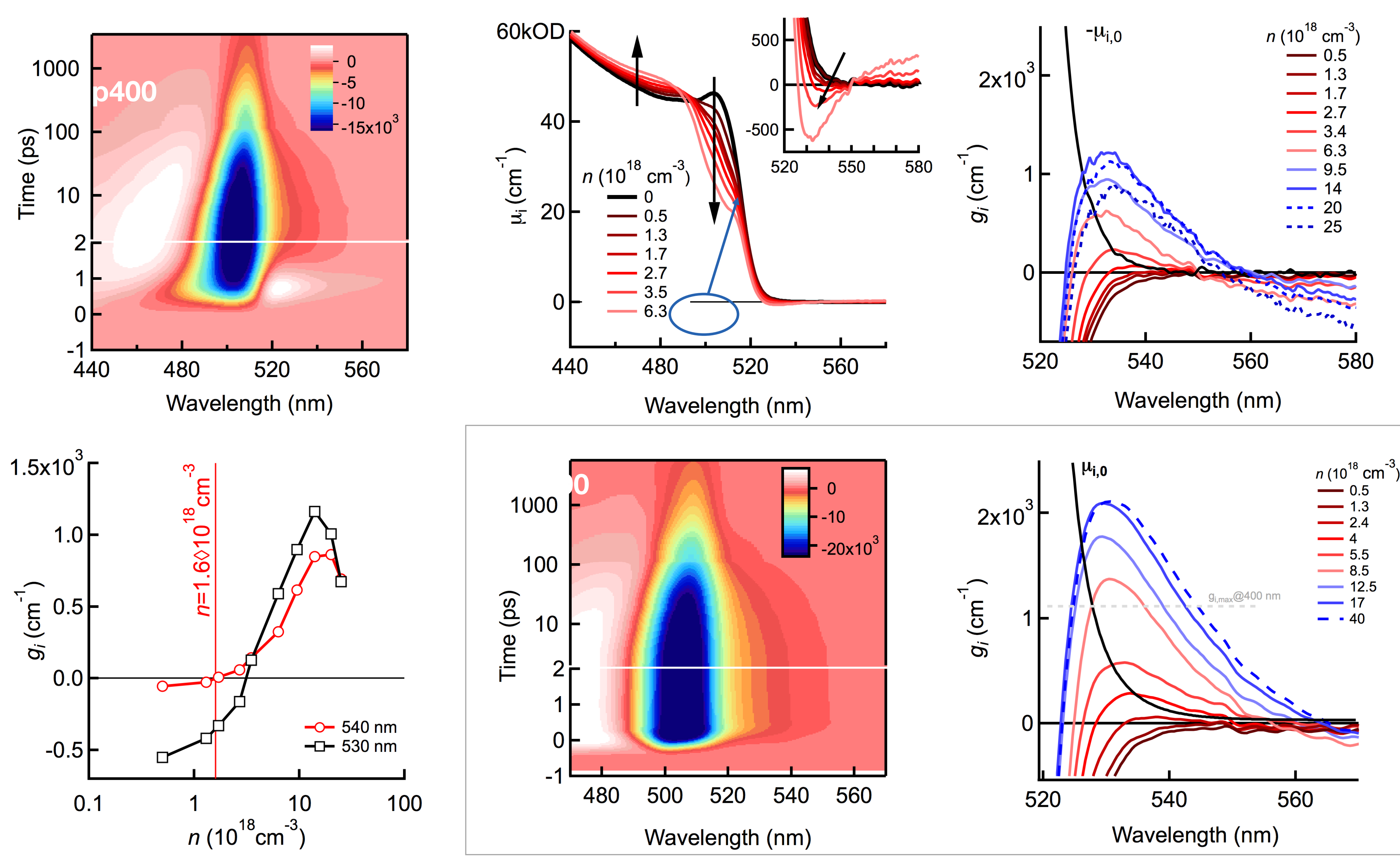

\section{Gain Threshold and Mechanism}

Bulk free-carrier gain threshold densities are observed, irrespective of NC size. Even at neardegenerate electron densities, a lingering exciton absorption counteracts broadband gain development..
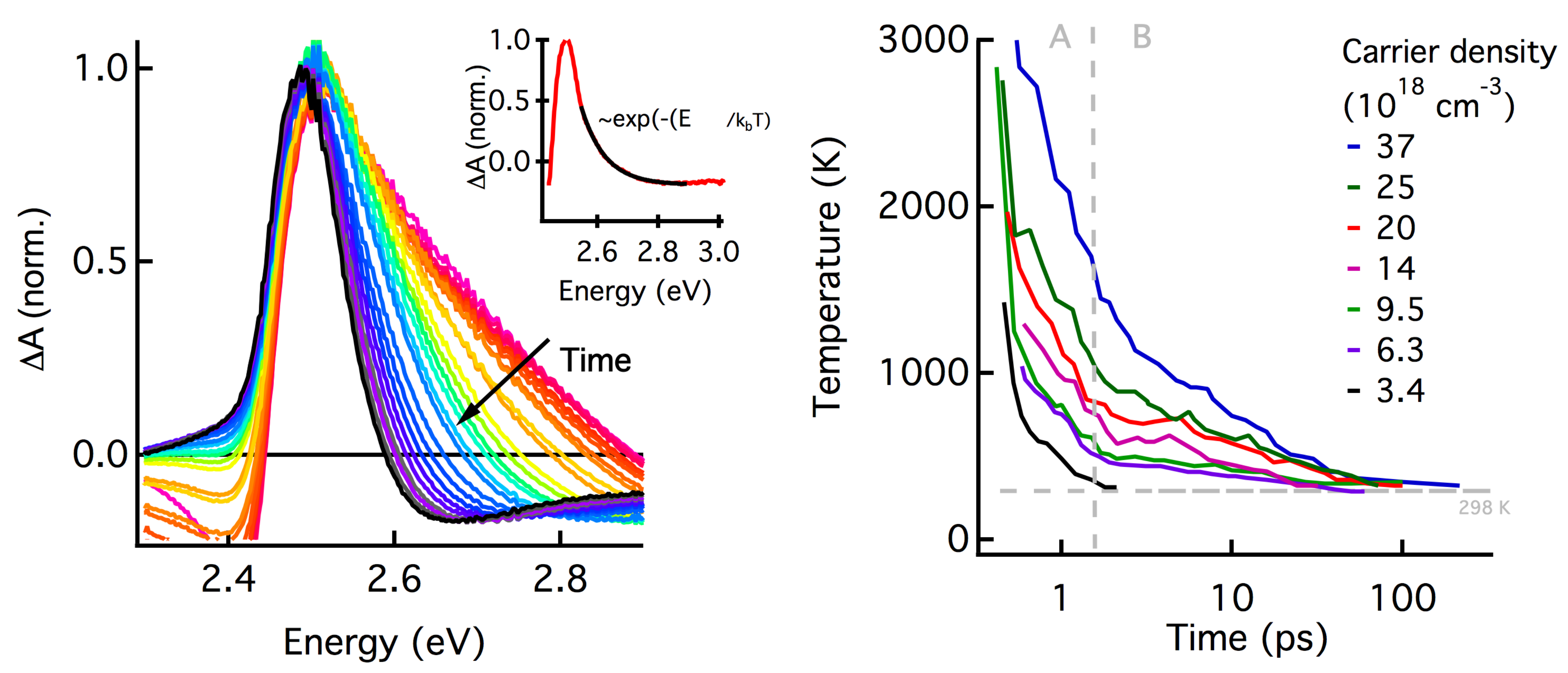